\title{
INDIRECT TRAUMATIC RUPTURE OF THE DIAPHRAGM
}

\author{
By H. D'Arcy Sutherland, M.S., F.R.C.S., F.R.A.C.S. \\ Honorary Thoracic Surgeon, Royal Adelaide Hospital, etc.
}

A survey of the literature on this subject reveals that many of the published works are based on isolated cases or small series. Collectively, the results are bad, because with their first cases many authors have either been unaware of the existence of the condition, failed to recognize it when it presented, or failed in the management when correctly diagnosed. The personal experience of the author, which is given in the following series of ten cases, includes all these errors. All the salient features have been encountered and the series is given in chronological sequence, so that the experience gained by ' trial and error' may be a guide to others.

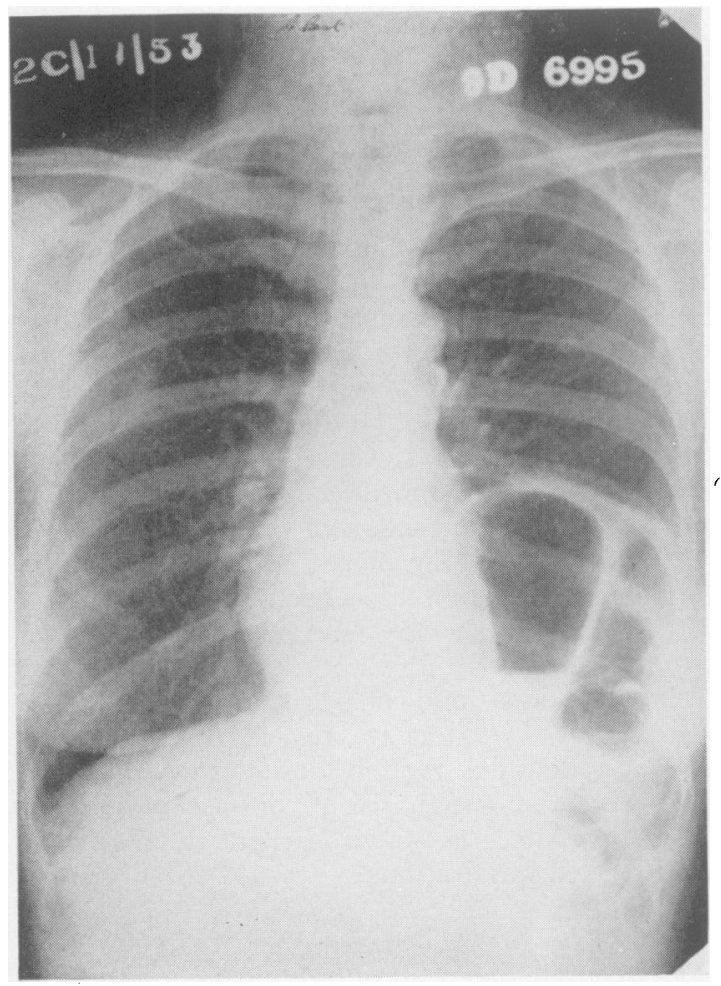

Fig. I

\section{Case I}

On April 28, I950, D.K., a male, aged 22, was admitted to the Royal Adelaide Hospital, following a head-on collision between two motor cars. He was complaining of pain in the left chest and left upper abdomen. He was shocked. There was diminished air entry and an impaired percussion note below the angle of the scapula on the left side. The abdomen was rigid with maximal tenderness in the left upper quadrant. He was observed as a possible case of ruptured spleen. His general condition steadily improved, but he had developed left shoulder tip pain when seen in consultation 


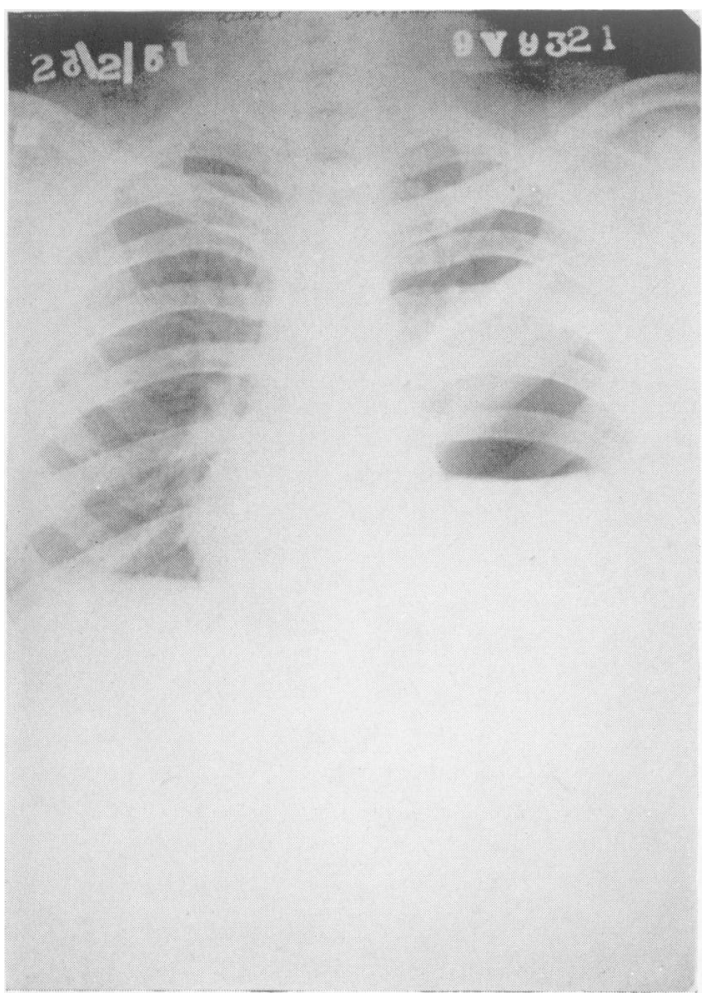

FIG. 3A

the next day. There was insufficient evidence for a diagnosis of ruptured viscus and conservative management was continued. An X-ray of the chest at that time (Fig. I) was reported as showing ' elevation of the left leaf of the diaphragm, suggesting paralysis of its fibres.' The significance of the X-ray appearance was not grasped. His condition improved rapidly and he was discharged for follow-up, but was temporarily lost sight of.

\section{Case 2}

Almost six months later, on October 22, I950, J.S., a female, aged 20 , was admitted with severe multiple injuries, as a result of a vehicular accident.

There was obvious respiratory distress. On examination of the chest the percussion note was impaired in the left axilla and there were no breath sounds on this side except at the extreme apex. The apex beat could be felt to the right of the sternum. The physical signs suggested a haemopneumothorax of some magnitude and an unsuccessful attempt made to aspirate the fluid. A mobile X-ray picture (Fig. 2 ) could only be taken in the supine position and this was reported as showing ' fractures of the 7 th, 8th, 9th roth and i ith ribs posteriorly and the left dome of the diaphragm is considerably elevated.' The possibility of rup-

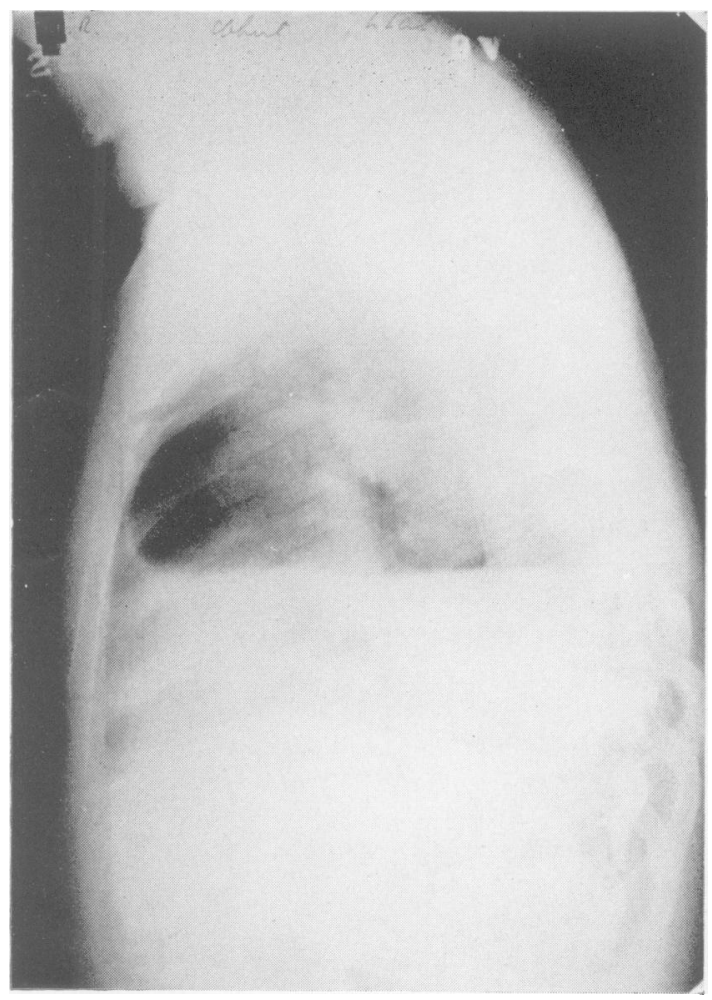

FIG. 3B

tured diaphragm was discussed when seen in consultation, but the patient's condition continued to deteriorate and she died in two hours, before any action could be taken. An autopsy revealed that together with many other injuries there was a laceration in the left leaf of the diaphragm, extending forward in the line of the muscle fibres from the region of the oesophageal hiatus for about $10 \mathrm{~cm}$. More than half the stomach was lying in the pleural cavity. It was then realized that the appearance interpreted as, 'elevation of the diaphragm,' was in fact due to the upper contour of the herniated and distended stomach, and the uniform opacity below this was caused by fluid in the stomach. There was no fluid level because of the supine position. It was also realized that the $\mathrm{X}$-ray appearance was similar to the undiagnosed lesion in Case I. Armed with this knowledge, Case I was traced through his local practitioner.

\section{Case I (continued)}

When seen again, he gave a history that since the accident he had become very short of breath on exercise and suffered attacks of vague collicky upper abdominal pain, particularly after sitting for long periods. The physical signs in the chest and $\mathrm{X}$-ray appearance had not changed since his 


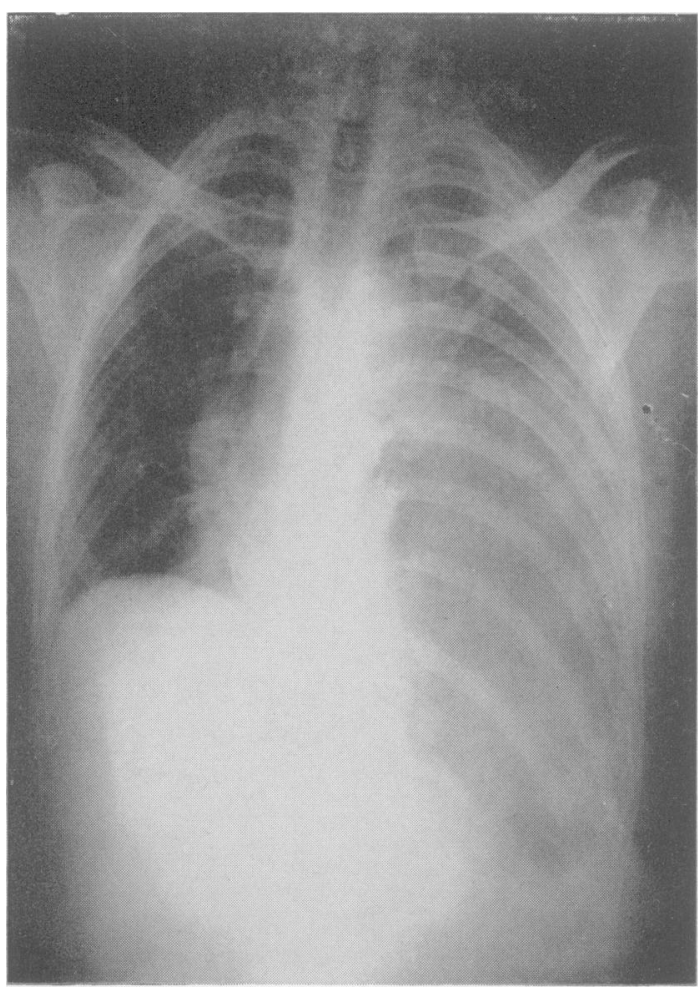

FIG. 4A

discharge from hospital. A pneumoperitoneum was performed and this confirmed the diagnosis, because the air immediately passed into the left pleural cavity. At thoracotomy through the bed of the left 8th rib, a linear tear was found extending across the left leaf of the diaphragm in the direction of its fibres, for a distance of $I 2 \mathrm{~cm}$. Its posterior limit was in the immediate vicinity of the oesophageal hiatus, nearly disrupting that structure. About half the stomach and the tip of the left lobe of the liver were in the thoracic cavity and held there by moderately dense adhesions. The abdominal viscera were reduced and restoration of the diaphragm was easily achieved by a Mayo type repair. When seen several months later, his exercise tolerance had returned to normal and he had been relieved of his abdominal pain.

\section{Comment}

The significance of the X-ray appearance was not appreciated when the case first presented. The patient passed rapidly into a quiescent phase, with vague but endurable symptoms. When eventually recognised, the condition was easily cured by operation.

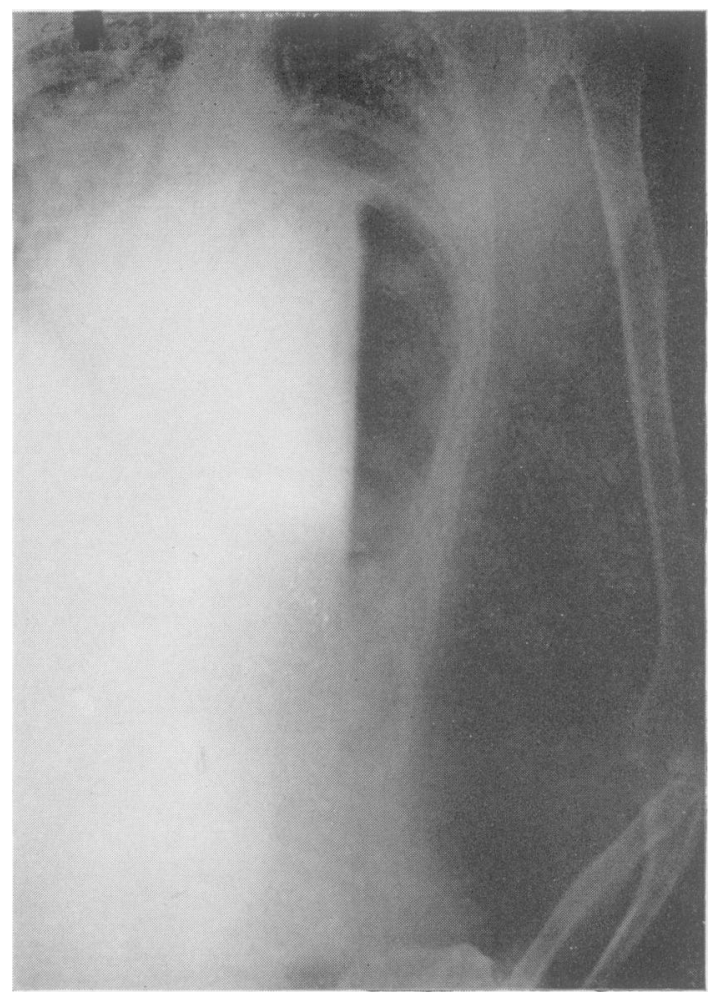

FIG. 4B

\section{Case 3}

On February 14, I951, R.W., a male, aged 22, was involved in a motor car accident, in which several of his fellow passengers were killed. His treatment began in the country, but he was transferred to the Royal Adelaide Hospital on February 24, I95 I, for treatment of a compound forearm fracture. Routine clinical examination on admission, revealed an impaired percussion note and diminished breath sounds at the base of the left lung. On further questioning, he stated that he had noticed some shortness of breath since the accident, but no pain in the chest or abdomen. A plain X-ray of the chest (Figs. 3 (a) and 3 (b)), revealed the typical appearance of stomach herniated through a tear in the left leaf of the diaphragm. At operation through the bed of the left 8 th rib the tear was found to have two limbs at right angles, one towards the pericardial adherence for about $5 \mathrm{~cm}$. from the main limb, which was ro $\mathrm{cm}$. long and extended from the centre of the dome, down to the left crus, but leaving the oesophageal hiatus intact. There was herniation into the chest of about two-thirds of the stomach and the tip of the left lobe of the liver. There were dense adhesions surrounding the displaced 


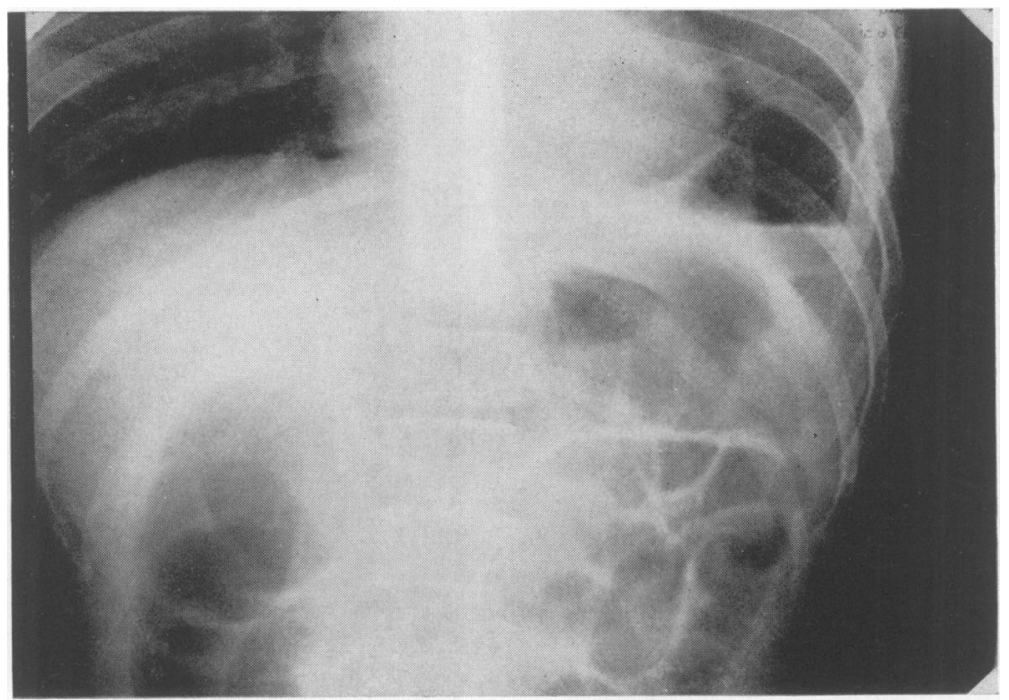

FIG. 5

structures, particularly to the torn margins of the diaphragm. After separation of the adhesinns, repair was easily effected without tension. The post-operative course was uneventful and he was discharged home on the IIth post-operative day.

\section{Comment}

This was similar to Case $I$, in that the patient passed into an almost symptomless, quiescent phase without the rupture being noted by his first medical attendants. Repair was easily effected by a transthoracic operation.

\section{Case 4}

On March 5, I95 I, R.S., a female, aged 68, was struck by a motor car and suffered injuries to her trunk and a compound fracture of a lower leg. On admission she was in considerable respiratory distress. She was cyanosed. There was gross displacement of the trachea and apex beat to the right, obvious diminution of movement of the left chest, no alteration of the percussion note, but breath sounds were not heard at the left base. A mobile X-ray of the chest (Fig. 4 (a)) revealed a number of fractured ribs (9th, Ioth and I Ith) and the appearance typical of ruptured diaphragm with gastric herniation. The diagnosis was confirmed by an antero-posterior film, taken in the right lateral position (Fig. 4 (b)), showing the fluid to be in the displaced stomach. The patient's general condition was poor and an attempt was made to improve her respiratory function by gastric suction, whilst intravenous fluids were given. Her condition improved to such an extent that repair of the torn diaphragm, which was being considered the next day, was postponed. She was resting without obvious pain and had only vomited once after admission. However, about 36 hours after admission, she complained of sudden severe pain in the chest, her condition rapidly deteriorated she became profoundly shocked and died within 12 hours. At autopsy, it was found that the tear in the diaphragm was adjacent to, but had not involved the circular fibres of the oesophageal hiatus and the gross distension of the body of the stomach had compressed the left gastric vessels across this like a constricting band. The fundus of the stomach was gangrenous and had ruptured into the pleural cavity.

\section{Comment}

The diagnosis was made on the clinical and chest $\mathrm{X}$-ray findings. Taking a film in the lateral decubitus position, confirmed the diagnosis (a helpful adjunct when barium studies are not practicable). The risk of strangulation of the stomach was not realized at this time and in retrospect it is considered that the patient was fit for operation 12 hours after admission. The patient became moribund too soon after the rupture of the gangrenous stomach to allow operation.

\section{Case 5}

On June 23, 1951, C.B., a male, aged 32, was admitted with a history of left-sided abdominal pain and vomiting of two-day duration. He stated that he had had several less severe attacks 


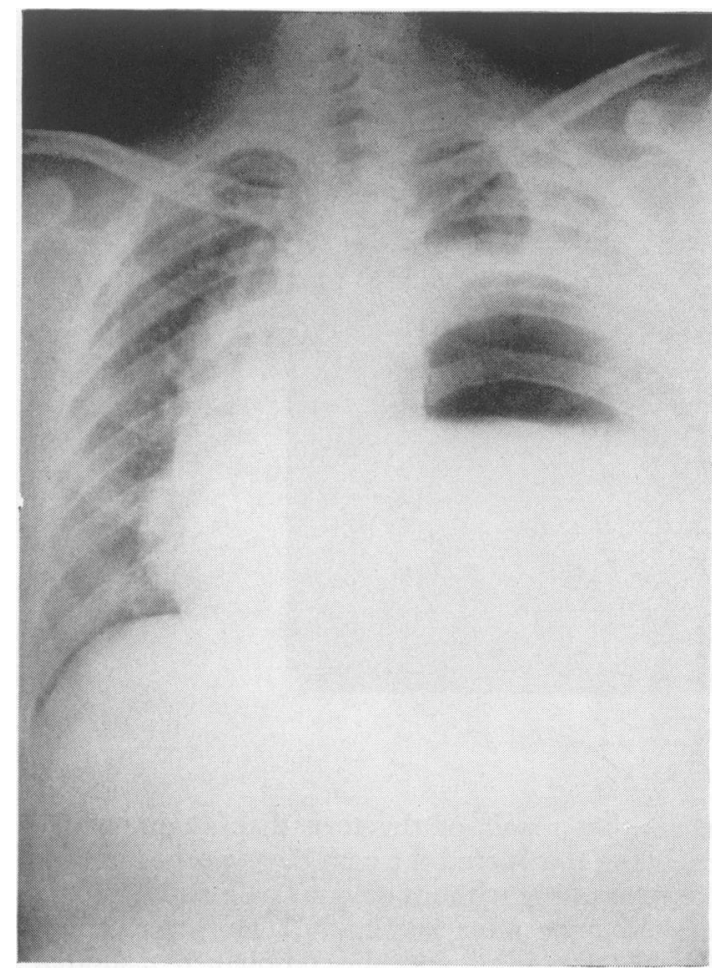

FIG. 6A

since a motor car accident four years previously. On that occasion, he was said to have suffered a left haemothorax and possible abdominal injury, but had recovered without operation. A clinical diagnosis of intestinal obstruction was made and a plain X-ray of the abdomen (Fig. 5), which included the diaphragmatic region, revealed the presence of a gas containing viscus above the normal level of the left diaphragm. A thoracoabdominal exploration was made forthwith by Mr. Alan Britten Jones, who found an old laceration of the diaphragm, extending from the dome towards the region of theoesophageal hiatus, through which a loop of colon and attached omentum had passed. The hernia was incarcerated and strangulation imminent. There was a little blood stained fluid in the pleural cavity. The combined approach facilitated the handling of the incarcerated tissues and the repair was performed from above. Recovery was uneventful.

\section{Comment}

The treatment following the vehicular accident was carried out elsewhere and no chest $\mathrm{X}$-ray was performed. The rupture of the diaphragm was

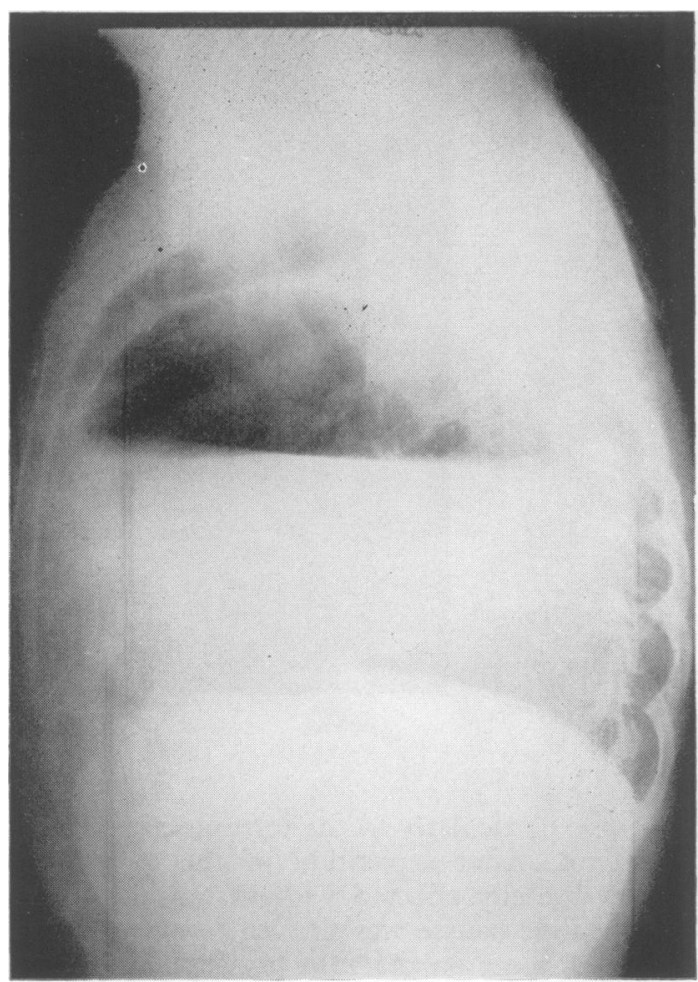

FIG. 6B

not diagnosed, nor was the significance of the repeated attacks of subacute intestinal obstruction appreciated. When incarceration occurred, an exact diagnosis was made by correct interpretation of the plain $\mathrm{X}$-ray examination and this resulted in an adequate surgical approach being planned. If the diagnosis had been made at a laparotomy, the manipulation would have been more difficult and the result may have been less successful.

\section{Case 6}

On November 5, I95I, A.K., a male, aged 33, was admitted with a history of severe upper abdominal pain radiating to the left chest, vomiting and hiccups of 40 hours' duration. He had been involved in a vehicular accident two and a half years previously and suffered a spinal injury, with resultant paraplegia. No chest injury was appreciated at the time and no chest $\mathrm{X}$-ray taken during his sojourn in hospital. On admission on the second occasion, he was cyanosed and severely shocked. The left side of his chest moved less than the right, was dull to percussion and without breath sounds up to the level of the angle of the scapula. There was clinical evidence of mediastinal shift to the right. His abdomen was not 


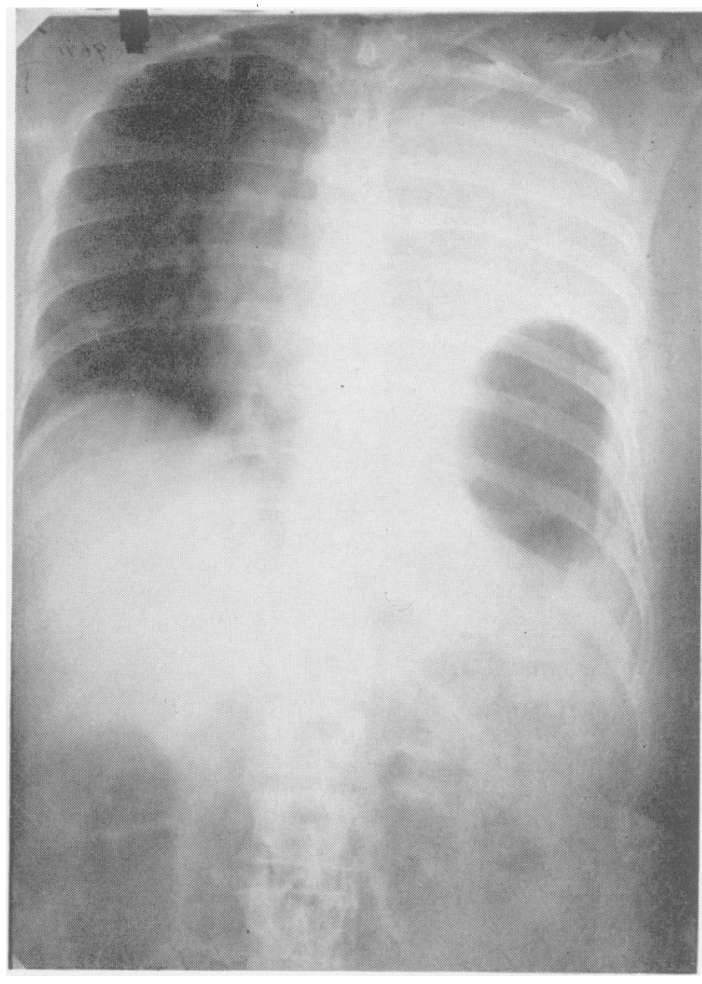

FIG. 7

distended. There was tenderness in the left upper quadrant, but no muscle guarding or rigidity. A plain X-ray film of the upper abdomen and chest (Figs. 6 (a) and 6 (b)) showed a large translucent area above the normal level of the left diaphragm with a crescentic upper margin with a fluid level across it. The X-ray also confirmed the gross mediastinal shift to the right. A Ryles tube was passed and $70 \mathrm{oz}$. of thin brownish fluid were aspirated and the patient's condition improved greatly. Gastric suction and intravenous therapy were continued for two days and his condition improved to such an extent, that mouth feeding was begun. Immediately after the first drink, he was smitten with severe pain across the left lower chest and his condition rapidly deteriorated. The case was first seen by the author at this stage. The clinical and radiological pattern was recognized and immediate operation planned, but the patient succumbed during the anaesthetic induction, one and a half hours later. An autopsy revealed a ruptured gangrenous stomach in the left hemithorax, complete collapse of the left lung and early empyema formation. There was a radial tear in the diaphragm from the region of the oesophageal hiatus, extending forwards to the dome. The acute angulation (as in Case 4), around the intact muscular ring of the oesophageal hiatus appeared to be the main cause of strangulation.

\section{Comment}

A case of ruptured diaphragm (unrecognized at the time of the accident) which passed into the quiescent phase and remained symptom free for two and a half years. When strangulation occurred, the clinical entity was not recognized until the patient became moribund. Except for the 'delay' of two and a half years, this case is similar to Case 4 , in that operation should have been undertaken shortly after admission to hospital.

\section{Case 7}

On May 17, 1952, E.D., a female, aged 56, was admitted to the Royal Adelaide Hospital, having been involved in a vehicular accident. Her only complaint was of severe pain in the left chest and shortness of breath. She was shocked. The apex beat was impalpable and heart sounds distant. The left chest moved less than the right. The percussion note was dull from the 12th rib, almost to the level of the spine of the scapula front and back, with absence of breath sounds in the same area. There was no evidence of abdominal injury. During the next two days, her general condition improved, but the dullness to percussion and absence of air entry extended almost to the clavicle. The X-ray appearance of her chest (Fig. 7) revealed a translucent area at the base above the normal level of the left diaphragm, suggesting traumatic herniation of the stomach and an extensive opacity above this (presumably fluid) the cause of which was not obvious. The case was referred to the Thoracic Surgical Unit at this stage and a provisional diagnosis of ruptured diaphragm made and thoracotomy performed the same day, through the left 8th rib bed. The pleural cavity contained a large quantity of blood and blood clot, which had come from a rupture of the spleen. The spleen had herniated into the pleural cavity with the stomach. The tear in the diaphragm was about $12 \mathrm{~cm}$. long, extending to the dome from the region of the oesophageal hiatus. The spleen was removed, the stomach replaced and the diaphragm restored by a Mayo type repair. The patient made an uneventful recovery.

\section{Comment}

This is the only case in the series with concomitant rupture of a viscus. The clinical and radiological picture was recognized as being caused by a rupture of the diaphragm with gastric herniation, but the significance of the extensive fluid collection was not appreciated until operation. 


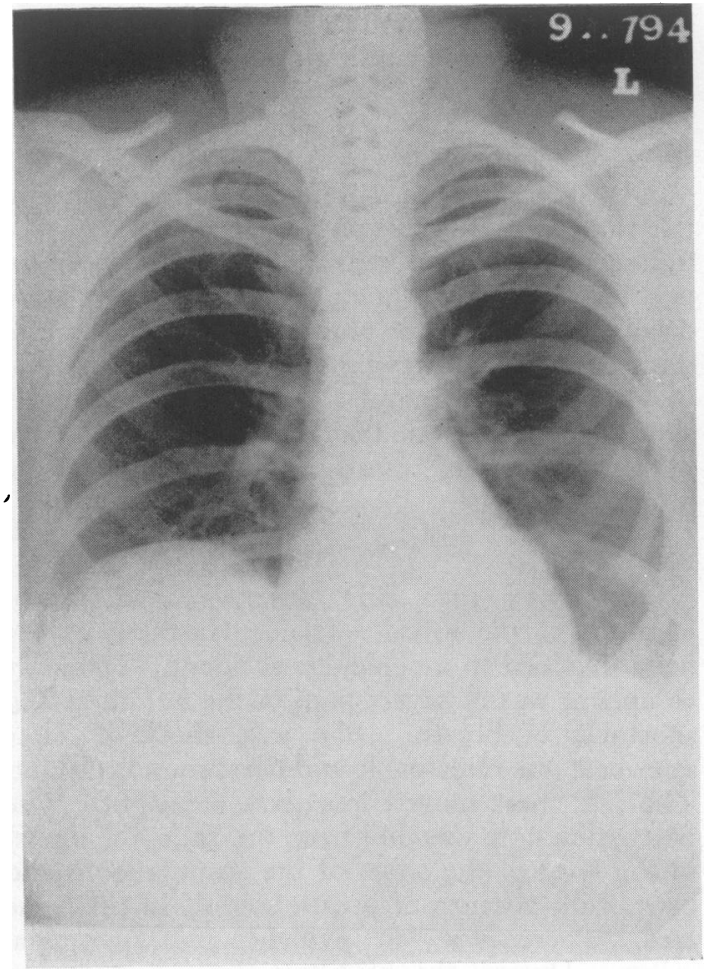

FIG. 8A

\section{Case 8}

E.J., a farmer's wife, aged 45 , was referred for treatment as a result of an X-ray survey film (Figs. 8 (a) and 8 (b)), which demonstrated a circular opacity in the right lower chest anteriorly. She complained of no symptoms, apart from a vague 'dragging' sensation in the right lower chest, which had been present since a severe vehicular accident ten years previously, in which she had suffered multiple fractures. The significance of the past history was not appreciated. In view of her rural habitat, she was operated on with a provisional diagnosis of hydatid cyst. At operation, there was an eliptical hole, about $8 \mathrm{~cm}$. in length, in the dome of the right leaf of the diaphragm in the line of its fibres, through which a mushroom-like herniation of liver had occurred. There were adhesions between the margins of the diaphragm and both the liver and the lung. Linear repair was effected and it was interesting that the patient claimed a significant increase in exercise tolerance post-operatively.

\section{Comment}

Lacerations of the right leaf of the diaphragm are rare. The true nature of the lesion could have been demonstrated by pneumoperitoneum, be-

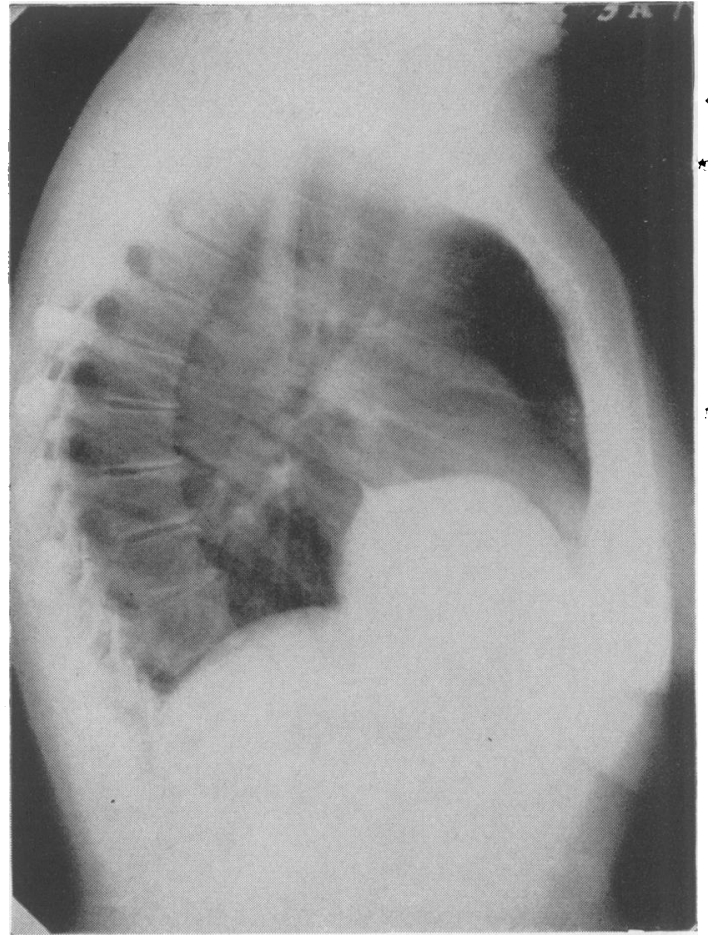

FIg. 8B

cause in spite of the adhesions there was a free passage between the abdominal and pleural cavities.

\section{Case 9}

On February 19, I954, a female, aged 60, was referred for treatment as a result of an X-ray survey film (Fig. 9) which had revealed the presence of a gas containing viscus, above the left leaf of the diaphragm. She gave a history of having been involved in a head-on motor car accident I9 years previously. Since that time, she had experienced ' windy pains' several times a week and occasionally experienced left shoulder tip pain after meals. Barium studies showed that the abnormal area contained a portion of the stomach and a loop of colon, which had herniated through an opening in the diaphragm, just anterior to its highest point. Operation was performed on March 2, I954, through an anterolateral 6th intercostal space approach. The lower half of the pleural cavity was obliterated by filmy adhesions. There was an old laceration in the diaphragm, extending from the infracardiac portion of the central tendon, almost to the anterior chest wall, about $8 \mathrm{~cm}$. from the midline. The laceration was $10 \mathrm{~cm}$. long. A loop of colon, 


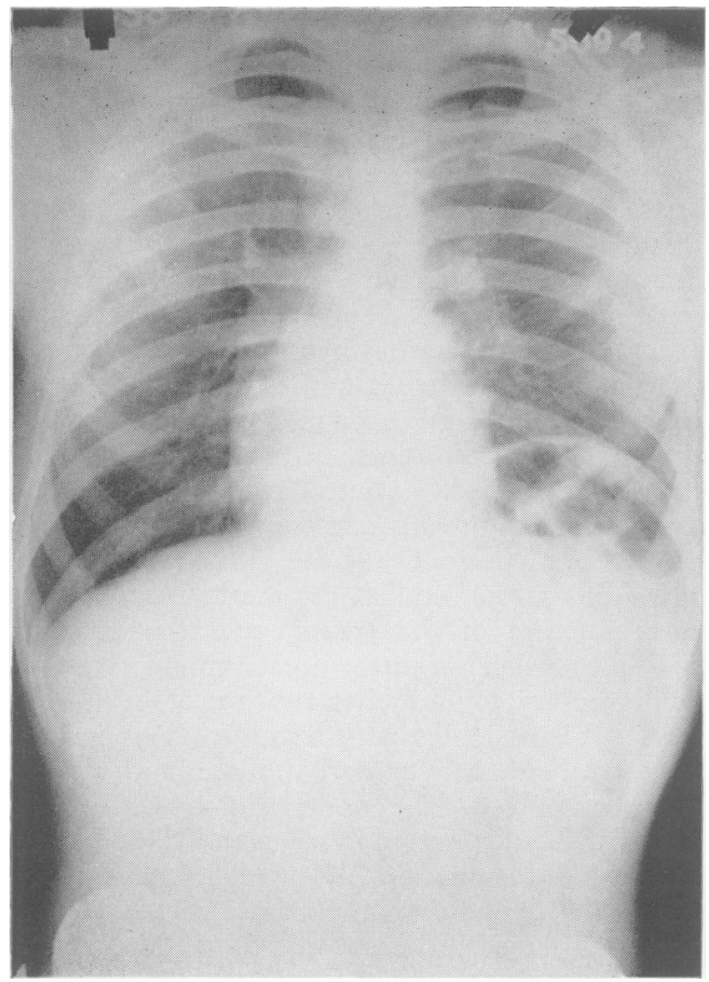

FIG. 9

omentum, the tip of the left lobe of the liver and a portion of the stomach had herniated through into the pleural cavity. Linear repair of the laceration was achieved without tension. Following operation, there was complete relief from the pain.

\section{Comment}

This case had remained in the quiescent phase for almost 20 years. However, in view of the fact that she was experiencing symptoms, suggestive of chronic partial obstruction, operation was recommended. This is an unusually long quiescent period, particularly with symptoms of this type. (Cf. Case 5, when strangulation occurred within three years; a period of time more consistent with that quoted in other published series.)

\section{Case ro}

On October 18, r955, R.T., a male, aged 7 years, was admitted to the Adelaide Children's Hospital, having been run across the midriff by a slow-moving truck. He was conscious, but could only speak with difficulty, owing to pain and respiratory distress. His face was suffused and

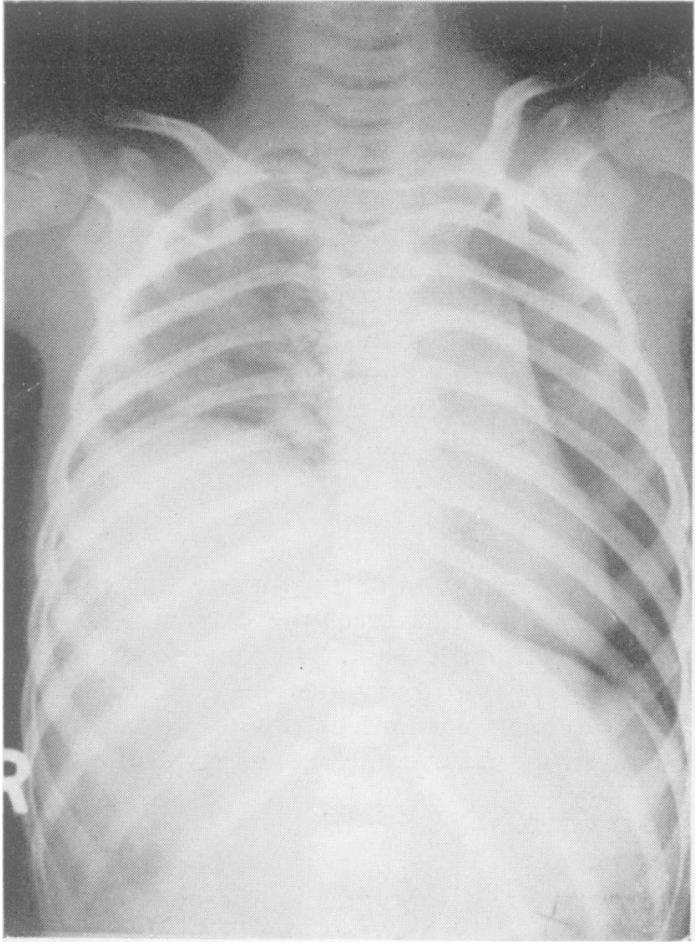

FIG. I0

there were petechial haemorrhages on his face, neck and conjunctivae. The left chest was apparently normal on clinical examination, but there was diminution of breath sounds at the right base and the liver dullness which began above the costal margin, extended to the third space anteriorly. His abdomen was not distended, rigid, nor unduly tender. He had a subluxation of the symphysis pubis and a fractured right lower leg.

An X-ray of the chest (Fig. I0) was reported as showing ' considerable elevation of the right leaf of the diaphragm' and the next day, there was evidence of pleural fluid. Over the next four days, a total of 270 c.c. of blood was aspirated from the right chest. His general condition improved steadily and was referred for treatment on 26th October, 1957. The X-rays showed that the whole liver had herniated into the chest through a large tear and that this was accompanied by some bowel. The operation had to be delayed because of an attack of chicken-pox, but was eventually performed on November I 5, I955. A thoracotomy was performed in the lateral position through the $7^{\text {th }}$ right intercostal space. There was a tear in the right leaf of the diaphragm, about $20 \mathrm{~cm}$. long, extending from the tip of the 7 th rib, along 
the periphery of the diaphragm to the anterior axillary line and then in an oblique direction posteriorly. The result being, that at least onethird of the periphery of the diaphragm was detached from the chest wall. The main substance of the diaphragm had retracted medially, the whole liver, portion of the transverse colon, some small bowel and omentum were lying in the pleural cavity. The diaphragm was re-attached to the chest wall, with a double row of chromic cat-gut sutures. Post-operative progress was ideal and when seen a year later, the X-ray of the chest was virtually normal in appearance and he was symptom free.

\section{Comment}

The only example of a slow crushing injury in the series. In this case, the extensive tear was repaired without tension. In some reports, repair of this type of injury has only been possible after resecting portions of ribs to allow the peripheral structures to come in to meet the diaphragm.

\section{Discussion \\ General}

Traumatic diaphragmatic hernia is usually considered under the two headings ' direct' and 'indirect.' The former refers to knife-wounds, shrapnel wounds, bullet wounds, erosion by drainage tubes, etc., and having many features in common with the latter, which forms the subject of this article. An increasingly common third group is now appearing in the literature in which the herniation results from disruption of a surgical incision in the diaphragm. Such cases are due to ' direct' trauma, but the usual site and size of the opening conforms more closely to that found in the 'indirect' group and consequently, so does the clinical course. An analysis of some of the larger series of traumatic diaphragmatic hernia reveals that, over all, penetrating wounds are the most frequent aetiological factor, particularly in the period after World War I. However, closed trauma to the trunk and crushing injuries are becoming of greater significance, as motor vehicular accidents increase.

\section{Aetiology}

The type of injury is usually some form of vehicular accident, severe blow in the midriff, a fall from a height, crushing by a fall of earth or being run over by a rubber-tyred vehicle. The compression of the abdominal contents causes a bu rsting force to act on the under surface of the diaphragm, which results in a linear tear in the line of the fibres from the region of the left central tendon towards the oesophageal ring, but rarely into it. Most of the reported cases follow this pattern, but there are variations with forked tears, extending into the right leaf or into the pericardium. The liver tends to protect the right leaf of the diaphragm and various writers quote the relative incidence on this side as only 5 per cent. of the left, but the incidence was 20 per cent. in this series. Strade and Vance in a review of the literature in 1953, could only find five cases of right-sided rupture from indirect trauma. At times, the tear detaches the diaphragm from the costal margin, as in Case 10, when the resultant cardiorespiratory disturbance is greater, but the risk of strangulation less, because a much greater opening is created. There are very few reports of associated rupture of a viscus and this occurred only once in this series (Case 7), when the spleen ruptured as it passed into the chest. In view of the severe nature of the trauma and the fact that multiple skeletal injuries are common, it seems contradictory to find that rupture of a viscus is rare. It is possible that if a rupture does occur, it may dissipate the explosive force and thereby reduce the effect of the compression by the time the dome of the diaphragm is reached. This seems a reasonable assumption, if one accepts that the disruption of the diaphragm is caused by a compression wave; because this must depend on intact abdominal viscera for its transmissi on The thoracic cage is rarely extensively damage $\mathbb{}$ and if any ribs are fractured, they are usuall below the level of the 8th ; this fact is also consistent with the force acting from the abdomen, rather than the chest. In no case, was there a pneumothorax, haemopneumothorax or surgical emphysema, one of which is usually present in severe injuries of the upper chest.

It seems therefore, that the force, at the instant of impact, must be applied solely to the under surface of the diaphragm.

\section{Symptoms and Signs}

The clinical picture is made up of a combination of the mechanical effect on the cardiorespiratory function by the displaced viscera and the pathological changes in the viscera themselves consequent upon their displacement.

The clinical entity is readily divided into three phases.

(a) That immediately following the trauma.

(b) A quiescent period.

(c) That associated with strangulation, if it occurs.

(a) The early symptoms and signs become confused owing to the multiplicity of injuries likely to be associated with this condition. The symptoms of pain in the left chest, pain in the left upper quadrant of the abdomen, left shoulder tip pain, shortness of breath, associated with shock 
and vomiting, are really non-specific. 'There is usually diminished air entry to a varying degree at the affected base and the percussion note may be impaired or unduly tympanitic. Tenderness and rigidity are common in the left upper quadrant of the abdomen. In none of the cases in this series was there good enough evidence to suggest peritoneal soiling.

The salient feature of this phase is the plain $\mathrm{X}$-ray of the chest, which gives an unmistakable picture, whether in the supine or erect position. The consistency of this picture has been demonstrated by all left-sided lesions in this series. It is considered therefore, that if the X-ray shows an appearance, suggesting 'elevation of the left leaf of the diaphragm' above its normal position, particularly if there is a large gas bubble and fluid level in the erect position, in a patient involved in the type of accident mentioned, the findings are pathognomic of ruptured diaphragm.

The sequence of events from this point varies considerably. The lesion may continue directly to strangulation with a false 'quiescent' period of only a few hours, as demonstrated by Case + or may pass into a true quiescent phase, which may last up to several years, as in Cases I, 3, 5, 6 and 9 . If strangulation follows immediately on the trauma, this is characterized by intractable vomiting. The vomitus may be of non-bile stained material or in the form of repeated haematemeses or merely dry retching. Severe pain developes in the left chest, left upper quadrant of the abdomen and left shoulder tip. The patient becomes profoundly shocked and rapidly moribund. The 'elevation of the diaphragm' and the size of the gas bubble are likely to be greater with strangulation than in non-obstructed cases. Carter has reported that the aspiration of sero-sanguinous fluid from the chest, when none was present before, is strongly suggestive of strangulation. It is of vital importance to recognize the onset of strangulation because if gangrene occurs, the mortality, even with operation, approaches 100 per cent., instead of being negligible, as it is in the absence of obstruction.

(b) It is more common for the lesion to pass into a 'quiescent ' phase with the response to treatment of shock. The improvement may be so rapid, that the medical attendant is completely hoodwinked only to be rudely awakened by the sudden onset of strangulation after hours, days, months or years. This rapid 'recovery' in spite of the constant danger of delayed strangulation, strengthens the case for performing a routine chest X-ray before discharge, on all persons suffering this type of trauma. If there is any doubt after plain X-ray examination and screening, barium studies will usually establish the diagnosis.
If the 'quiescent' phase is prolonged, there may be no symptoms or only vague symptoms, such as shortness of breath on exercise, substernal discomfort after meals, vague upper abdominal discomfort, sometimes relieved by vomiting or attacks or subacute intestinal obstruction. These symptoms may be insufficient to make the patient seek medical attention.

(c) The onset of strangulation is said to occur most commonly within three years of the injury and its onset is usually sudden. It may follow raised intra-abdominal pressure due to unwonted straining and cases have been reported as coming on in the later months of pregnancy. In many cases the existence of the traumatic hernia is unsuspected (Cases 5 and 6 ) and the history of a previous accident may not be volunteered. 'The presence of intestinal obstruction is probably recognized, but in the absence of abdominal distension and with the bizarre pattern of upper abdominal, chest and shoulder tip pain, conservative management may be continued too long (Case 6). A 'wait and see' policy may be encouraged by improvement after the passage of a Ryles tube (Cases 4 and 6) or the obtaining of encouraging returns from enemata. If gangrene occurs, the outlook is almost hopeless. In Cases 4 and 6 , the compression of the left gastric vessels by the intact oesophageal ring, appeared to play a major part in the causation of gangrene, but in Case 5 the strangulation was of the type more commonly associated with ' direct ' diaphragmatic hernia when the opening is smaller. In Case 6, the significance of a plain X-ray, which made the diagnosis certain, was not grasped until too late; whereas in Case 5, a correct interpretation was made and immediate operation forestalled the onset of gangrene.

Rare cases have been reported with a 'normal' $\mathrm{X}$-ray immediately after admission, but in whom herniation and even strangulation occurred later. It is presumed in these circumstances that omentum or the spleen temporarily covered the opening, but raised intra-abdominal pressure later caused the herniation.

The picture presented on the right side may be quite different from that on the left, in that bowel is rarely herniated into the chest unless the tear is large (as in Case Io) and consequently the risk of strangulation is minimal. The symptoms of cardiorespiratory dysfunction are more in evidence because of the gross displacement of lung, the paradoxical movement of the viscera through the large opening and the gross loss of function of the right half of the diaphragm.

\section{Diagnosis}

At some stage in the natural history of this 
condition, the diagnosis will be obvious and if this stage is only reached at the autopsy, it becrmes painfully so.

In the immediate post-traumatic stage, the possibility of ruptured diaphragm should be considered on clinical grounds alone, if after a severe vehicular accident, fall from a height or crushing injury, there are signs of reduced air entry at the base of one lung, with dullness or tympany in the same area, mediastinal shift minimal evidence upper rib damage, disproportionate respiratory distress and intractable vomiting. These findings may rightly be considered too nebulous to make the diagnosis, but at least they are sufficient to justify a chest X-ray on which the diagnosis can be made almost for certain. The typical appearance of the 'elevated diaphragm' with the large stomach gas bubble and mediastinal shift to the opposite side have been amply demonstrated by this series. Barium studies make the diagnosis certain. However, if the patient is too ill for these to be carried out, an antero-posterior mobile chest X-ray in the right lateral decubitus position is almost as convincing (Fig. 4 (b)).

In the quiescent phase the diagnosis will only be entertained if the vague symptoms of shortness of breath, upper abdominal discomfort, substernal discomfort after meals, recurrent partial intestinal obstruction, etc., are related in the history to some accident. Alternatively if plain X-ray of the chest and barium studies are being carried out because of the above symptoms, these should make the diagnosis a relatively simple one. If the possibility of congenital eventration of the diaphragm is being considered, the performance of a pneumoperitoneum and $\mathrm{X}$-ray in the upright position should rule this out, because with a ruptured diaphragm, the air passes directly to the pleural cavity and in congenital eventration, it lies between the abdominal viscera and the elevated diaphragm.

After the late onset of strangulation, the history of trauma is rarely given by the patient and the bizarre symptoms of chest, shoulder tip, as well as upper abdominal pain, plus the relief from decompression of the stomach by Ryles tube, all make for an undue delay, which may prove fatal. If the stomach is the only viscus strangulated, the clinical picture is clearly that of alimentary obstruction, but the complete absence of abdominal distension with the chest and shoulder tip pain, should direct attention to the region of the diaphragm. The presence of blood in the vomitus and an increase of sero-sanguinous fluid in the pleural cavity, are both said to be indicative of the onset of strangulation. If the stomach is strangulated around an intact oesophageal hiatus, the Ryles tube may not pass into the stomach and if a barium study is possible, this will show a complete block at this point.

\section{Treatment}

Surgical treatment is the only possible solution and as the above series shows that this should be considered an urgent necessity in all stages, both to obviate the possibility of strangulation and to restore to normal the impaired cardio-respiratory function. There is no justification for delaying operation, even a matter of hours, in the immediate post-traumatic phase, once shock is sufficiently controlled. The death of Case 4 could have been avoided if one had not been mislead by the 'improvement' with intravenous therapy and gastric suction.

In the quiescent phase, operation is almost as urgent because the onset of strangulation can occur at any time.

There is no need to stress the need for operation if strangulation does occur. Under normal circumstances, the best surgical approach is through the chest, at the level of the 8th rib, but if strangulation has occurred, the incision should be planned as a thoraco-abdominal one for more adequate exposure and for easier access to the bowe particularly if the colon is involved. Even thougto this condition has been successfully manage through an abdominal incision in the past, authors are now almost unanimous in advocating an 8th rib transthoracic approach. The repair of the diaphragm once the adhesions are separated, is easily achieved and there is usually sufficient tissue to make a Mayo-type repair. If the tear destroys the musculature of the oesophageal hiatus, this should be carefully reconstituted. When the diaphragm is avulsed from the chest wall and there is insufficient tissue left peripherally repair should be effected by suturing the free edge to the chest wall with interrupted sutures to two adjacent intercostal muscles to obtain a wider adherence. In the opinion of the author, there is no justification for paralysing the phrenic nerve, even by ' temporary' crushing, but on rare occasions, it may be necessary to resect lengths of the lower ribs subperiosteally, to allow closure of a peripheral tear. The thoracic cavity should alvays be drained by an underwater seal system.

\section{Prognosis}

If the patient recovers sufficiently from the trauma to undergo operation, and if strangulation has not occurred, the results are universally good. The onset of strangulation increases the morbidity and mortality and with the onset of gangrene, the outlook as demonstrated in all published series, is 
almost hopeless and very few recoveries have been recorded.

One of the first cases recorded in the English literature, was by Wright, in I935, and his concluding remarks are most appropriate. 'It would appear that routine visualization of the stomach by barium is desirable in all cases of crushing injuries to the chest, if the surgeon is to receive the case early and not when some catastrophe such as acute strangulation has rendered his help imperative, but has vitiated the chance of a successful outcome to a difficult operation.'

\section{Summary}

A personal experience of ten cases of indirect rupture of the diaphragm with closed trunk injuries is presented.

The case histories are given in chronological order and in some detail because as well as illustrating the features of the condition, they give a good account of the author's education in its management.

It is considered that the most important single factor in making the diagnosis, is the recognition of the plain chest X-ray appearance. Once this is understood, the diagnosis will rarely be missed.

With this in mind, a plea is made for early routine plain chest X-ray of all cases following severe trauma to the trunk, particularly those with midriff pain and signs of reduced function of one or other lung base.

Surgical treatment is clearly indicated as a matter of urgency in all cases, because of the high mortality if gangrene has occurred, compared with a negligible one before strangulation.

\section{Acknowledgements}

To the physicians and surgeons of the Royal Adelaide Hospital and Adelaide Children's Hospital, who referred cases for treatment.

To Mr. Alan Britten Jones, for permission to include his case which was only seen by the author in consultation.

\section{BIBLIOGRAPHY}

BUGDEN, WALTER F., et al. (1955), Ann. Surg., 142, 851 CARTER, B. NOLAND, et al. (195I), Amer. Y. Roentgenol., 65, 56 CARTER, B. NOLAND, and (GINOEFFI, J. (1948), Ann. Sirg.

I28, 2 IO.
DUGAN, D. J., ard SAMSON, P. C. (1948), f. thorac. Siurg.,

HAMIL 77Ó. 78, 686

HARRINGTON, S. W. (1945), Ann. Surg., 122, 546.

HARRINGTON, S. W. (1933), F. Amer. med. Ass., ror, 987 HEDBLOM, C. A. (1925). Ibid., 85, 947.

MANLOVE, C. H., and BARÖNOFSKY, I. D. (1955), Surgery, $37,461$.

MARSHALL and SANFORD, et al. (1956), Postgrad. med. Y., I9, 60

SCHNEIDER, CHARLES F. (I956), Amer. F. Surg., 91, 290

STRODE, F. C., and VANCE, C. A. (1953), Ann. Surg., 137, foo. IVRIGHT, R. D. B. (1935), Brit. med. $\mathcal{Y}$., ii, 577 .

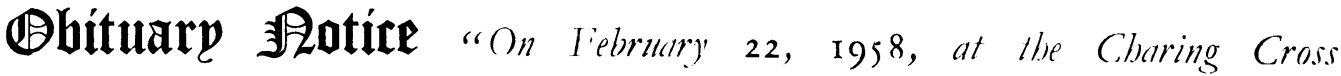
Hospital, Toby' Levitt, M.A.(Cape Ton'n), I'.R.C.S.ling., passed an'(!)' peacefully' after a long illness, bravely evidured."

It is with regret that we record the death of Dr. I evitt, who did a great deal of work for the Fellowship, and whose demonstrations and lectures were universally appreciated by those who attended his classes. Our sympathy is extended to his sister.

Continued from page 209--Subclavian Lymph Node by' Cotter Harrey and Peter Harvey.

tinal disease may necessitate consideration of biopsy procedure.

2. Three possibilities are discussed:

(a) Scalene node biopsy, especially for widespread pulmonary infiltrations and mediastinal adenomegaly.

(b) Pleural biopsy for pleural effusions.

(c) Lung biopsy for diffuse pulmonary infiltrates otherwise undiagnosable.

3. 'These procedures have minimal morbidity and they merit more widespread use.
4. Cases are reported to illustrate the value of these methods.

\section{REFERENCES}

. DANIELS, A. ('. (1949), Dis. Chest, 16, 360.

2. SHEFTS, L. M., et al. (1953), Amer. Rev. Tuberc., 68, 505.

3. CARSTENSEN, B., et al. (1954), Dis. Chest., 25, 443.

4. SOTCLIFFE, W. D., et al. (1954), Ibid., 26, 551 DE FRANCIS, N., KLOSK, F., and ALBANO, E., N. Eng. f. Med., 252, 998, 955

6. FLESHMAN, S. J., et al. (1956), Thorax, 11, 324

7. KLASSEN, K. P., et al. (1949), Arch. Surg. (Chicago), 59, 694 9. EFFLER, D. B., et al. (1955), Amer. Rev. Tuberc., 71, 668.

9. THEODOS, P. A., et al. (I955), Dis. Chest, 27, 637.
Io. STOREY, (. F., and REY'NOLDS, B. M. (I953), Ibid., 23, 357 